\title{
Holographic Abelian Higgs model and the linear confinement
}

\author{
Eunseok $\mathrm{Oh}^{*}$ and Sang-Jin $\mathrm{Sin}^{\dagger}$ \\ Department of Physics, Hanyang University, Seoul 04763, Korea
}

(Received 18 November 2018; accepted 18 February 2020; published 24 March 2020)

\begin{abstract}
We consider the holographic Abelian Higgs model and show that, in the absence of the scale symmetry breaking effect, chiral symmetry breaking gives linear confinement where the slope is given by the value of the chiral condensation. The model can be considered either as the theory of superconductivity or as the axial sector of QCD depending on the interpretation of the charge. We also provided a few models with linear confinement.
\end{abstract}

DOI: 10.1103/PhysRevD.101.066020

\section{INTRODUCTION}

One of most spectacular phenomena provided by the strong interaction is the stringy structure out of systems of particles, and the leading guidance in strong interaction has been the chiral symmetry. The string theory was born by the observation [1,2] that a linear relation between the mass squared and spin in the data of hadrons, the Regge trajectory, can be realized by the spectrum of a vibrating string. Naturally, in the era of the AdS/CFT [3-5], two of the leading questions in its application were how to utilize the chiral symmetry $[6,7]$ in the new context and whether holography can produce the linear trajectory for QCD.

When the vacuum expectation value of $\bar{q} q$ is nonzero, chiral symmetry is broken, and QCD is in the confining phase. Therefore, these two are likely related, and therefore linear Regge trajectory and the chiral symmetry breaking should be so also. However, this has not been so clear even in the anti-de Sitter (AdS)/QCD era, although the linear spectrum for the vector meson sector was given in Ref. [7] using a dilaton configuration.

In this paper, we will point out that the holographic Abelian Higgs model has a linear confining spectrum. That is, the chiral symmetry breaking is enough to establish the Regge trajectory in the axial sector of the QCD. We consider the one flavor case for simplicity so that the theory becomes an Abelian gauge theory. Notice that even for the multiflavor case, the nonlinearity due to the nonAbelian structure is irrelevant in leading order discussion

\footnotetext{
lspk.lpg@gmail.com

†sangjin.sin@gmail.com
}

Published by the American Physical Society under the terms of the Creative Commons Attribution 4.0 International license. Further distribution of this work must maintain attribution to the author(s) and the published article's title, journal citation, and DOI. Funded by SCOAP ${ }^{3}$. on the spectrum and transports, which ensures that the linearity of the spectrum remains for the multiflavor case.

The left- and right-handed quarks, $q_{L}, q_{R}$, have axial charge -1 and +1 respectively under the axial $U(1)$ global symmetry. Invoking the holographic principle, we have axial gauge field $A_{\mu}$ and a complex scalar field $\Phi$ which is the dual to the $\bar{q}_{L} q_{R}$ of charge 2 . Because the presence of the pion indicates that the chiral symmetry is broken spontaneously, so is the promoted chiral $U(1)$ gauge symmetry. Thus, we are naturally lead to the Abelian Higgs model where $\Phi$ plays the role of Higgs field. We will show below that this model has the linearly confining spectrum.

On the other hand, we will see that when there is a dilatonic effect like in the soft-wall model [7], spontaneous chiral symmetry breaking will not give any significant contribution to the Regge slope; instead, it can contribute to the Regge intercept. Namely, the Regge slope is predominantly determined by the gluon condensation only, and this explains why all the Regge slopes are the same. This can be traced back to the fact that the complex scalar field is dominated by the dilaton configuration in the infrared regime. Therefore, in this paper, we do not use overall dilaton factor $e^{-\varphi}$, and will give a phenomenological QCD model where spontaneous chiral symmetry is not disturbed by dilaton and linear confinement is respected in all sectors.

We will also give a various models with linear confinement properties which may and may not be related to the real QCD, because our observation on the stringy spectrum is very universal, not necessarily attached to the phenomena of QCD.

\section{STRINGS IN HOLOGRAPHIC ABELIAN HIGGS MODEL}

We start from the canonical action of the gauge field $A_{\mu}$ and the complex boson $\Phi$ in a fixed metric background. 
$S=\int d^{d+1} x \sqrt{-g}\left(-\frac{1}{4} F_{\mu \nu}^{2}-\left|D_{\mu} \Phi\right|^{2}-m_{\Phi}^{2}|\Phi|^{2}\right)$,

where $D_{\mu}=\nabla_{\mu}-i q A_{\mu}$ is the covariant derivative in the $\operatorname{AdS}_{d+1}$ of radius $L=1$ whose metric is

$$
d s^{2}=\left(d z^{2}+\eta^{\mu \nu} d x^{u} d x^{\nu}\right) / z^{2}, \quad \text { with } \eta^{00}=-1 .
$$

Bulk mass $m_{\Phi}^{2}$ is given in terms of the conformal dimension of the dual operator: $m_{\Phi}^{2}=\Delta(\Delta-d)$. We will fix it such that $\Delta=2$, which is natural in $d=2+1$ dimensions. For $d=3+1$, we need to choose $m_{\Phi}^{2}=-4$ for $\Delta_{\bar{q} q}=2$, which is realized at the left boundary value of conformal window of $N_{f} / N_{c}$ [8]. Since we are applying the AdS/CFT in the confined phase at the low energy where the conformality is lost, the boundary value 2 is better than the free fermion value 3 . The field equation then gives

$$
\begin{aligned}
& \Phi=M_{0} z+M z^{2}, \quad \text { in } \mathrm{AdS}_{4}, \\
& \Phi=M_{0} z^{2} \ln z^{-1}+M z^{2}, \quad \text { in } \mathrm{AdS}_{5},
\end{aligned}
$$

which are exact solutions of the scalar field equations in the probe limit. Since we look for the dynamically generated gap, we set the source $M_{0}=0$ so that $\Phi=M z^{2}$.

Now, the Maxwell equation is given by

$$
\nabla^{\mu} F_{\mu \nu}=J_{\nu}
$$

and for the real solution of $\Phi$, the current is simplified to the London equation similarly to the superconductivity,

$$
J_{\mu}=q^{2} \Phi^{2} A_{\mu} .
$$

For the transverse components with $\vec{k} \cdot \vec{A}=0$, it can be rewritten as Schrödinger equation [7] via $\Psi=\frac{A_{x}}{z^{(d-3) / 2}}$,

$$
\begin{gathered}
-\Psi_{n}^{\prime \prime}+V \Psi_{n}=E_{n} \Psi_{n}, \\
V=\frac{p^{2}-\frac{1}{4}}{z^{2}}+q^{2} M^{2} z^{2} \\
E_{n}=q M(4 n+2 p+2),
\end{gathered}
$$

with $p=(d-2) / 2$, and $E_{n}=\omega^{2}-k^{2} \equiv m_{n}^{2}$. The corresponding wave functions are given by

$$
\Psi_{n}(z)=N e^{-\frac{1}{2} q M z^{2}} z^{p+\frac{1}{2}} L_{n}^{p}\left(q M z^{2}\right),
$$

where $N=\sqrt{\frac{2 n !(q M)^{p+1}}{(n+p) !}}$. The mass spectrum is

$$
m_{n}^{2}=4 q M(n+d / 4) .
$$

For tensor with rank $s$, there are a few possible models according to the permutation symmetry of the index and gauge symmetry of the theory. As we have shown in the Appendix, some of them have spectrum

$$
m_{n, s}^{2}=4 q M\left(n+s-1+\frac{d}{4}\right) .
$$

For $d=4$, these results coincide with those of Ref. [7], where vector meson spectrum was discussed using the dilaton. Notice that here we did not use the dilaton. The reason for such a coincidence is just because the equations of motion of the two models turns out to be the same when they are expressed in Schrödinger form in spite of the difference in the degree of freedom. However, this is because we use the scalar solution in the probe limit at zero temperature. When we consider the effect of the finite temperature or backreaction or chemical potential, the difference will be manifest. For $d=3$, the $1 / z^{2}$-potential is accidentally canceled, but the spectrum is still given by the above formula because we need to impose the boundary condition $A_{\mu}=0$ at the boundary of AdS.

For general spin $s$, we need to choose the mass term of the higher spin fields properly to get Eq. (12). That spin dependent mass is necessary for the spectral formula has been known from the original paper [7] but has not been so clear. Notice that in string theory, the action encodes all the spin simultaneously, while in field theory, the action for each spin should be considered one by one. Now, how does one add up such spin dependent field theories to describe the holographic image of the bulk fundamental string? While the kinetic terms are canonical, the mass term and interaction term of spin $s$ excitation are ambiguous. We suggest that reproducing the linear spectrum can be used as a guiding principle to determine them especially if our purpose is to describe a theory whose spectrum follows Regge trajectory. Then, the statement is that, for any spin of given symmetry, there exist a choice of mass term such that the resulting spectrum is given by Eq. (12).

Notice that the spectrum is linear in both spin $s$ and vibrational quantum number $n$, and therefore the model has a spectrum of the open string whose string tension is

$$
T=1 /\left(2 \pi \alpha^{\prime}\right), \quad \text { with } \alpha^{\prime}=1 /(4 q M) \text {. }
$$

In the $M \rightarrow 0$ limit, that is, in the tensionless limit, the whole tower of the string spectrum is reduced to that of a massless particle.

Although we considered the only Abelian theory, the same spectrum will be obtained for non-Abelian theories. This is because holographic spectrum analysis depends only on the quadratic part of the field variation's action. Therefore, when we consider SU(N) and we perturb around 0 background gauge field, the nonlinear terms induced by the non-Abelian-ness do not affect the spectrum. Therefore, 
nonlinear chiral dynamics in holography will also give the linear spectrum. That is, our spectrum is the same as that of the $2+1$-dimensional version of the model studied in Ref. [6] if the boundary condition is the same. In fact, the important difference is soft-wall boundary condition (BC) installed by scalar condensation. The authors of Ref. [6] did not get the linear spectrum because they assumed the hard wall BC. In Ref. [7], the authors introduced the soft wall by hand using the dilaton dressing which is not supported by an equation of motion. Then, in terms of the QCD, what we did was use the action of the hard-wall model but install the soft-wall BC by scalar condensation.

So far, we have seen that the Abelian Higgs model considered as the axial part of the QCD has a linear spectrum. In our field theory description, we have seen that for each spin $s$ excitation of the string in AdS, which we called "spin $s$ particle in AdS," creates a tower of the linear spectrum in the boundary.

\section{TWO COMPETING ORDERS IN QCD: SCALE AND CHIRAL SYMMETRY BREAKINGS}

So far, we have not considered scale symmetry breaking. We now consider how including it can change the behavior of the theory. In fact, one of the important mechanisms of mass generation in the QCD is the scale symmetry breaking. The dilaton field has been usually considered as the dual of the gluon operator. In Ref. [7] of the soft-wall model, the dilaton factor $e^{-\varphi}$ was used without specifying the origin. In this paper, we identify $\varphi$ as the square root of the gluon operator: $\varphi^{2} \sim \operatorname{Tr} F_{\mu \nu} F^{\mu \nu}$. Since we do not want to modify the AdS metric in the Einstein frame, we determine the dilaton in the AdS background. Then, $\varphi$ is of dimension 2 , and we can write down the bulk action of it using the bulk mass $m_{\varphi}^{2}=-4$. Setting the source part of $\varphi$ to be zero as before, we get

$$
\varphi=G z^{2}, \quad \text { with } G^{2}=\left\langle\operatorname{Tr} F_{\mu \nu} F^{\mu \nu}\right\rangle .
$$

Now, the action of the soft-wall model is

$$
S=-\frac{1}{4} \int \sqrt{g} e^{-\varphi}\left(F_{A}^{2}+F_{V}^{2}+4\left|D_{A} \Phi\right|^{2}\right) .
$$

The Schrödinger form of the equation of the transverse component of $V_{i}, A_{i}$ is $-\psi^{\prime \prime}+V \psi=m_{n}^{2} \psi$ with

$$
\begin{aligned}
V & =G^{2} z^{2}+\frac{3 / 4}{z^{2}} \quad \text { for vector } V_{i}, \\
& =G^{2} z^{2}+\frac{1+|\Phi|^{2}-1 / 4}{z^{2}}, \text { for axial vector } A_{i},
\end{aligned}
$$

where $\psi=\mathcal{A}_{\perp} /\left(\sqrt{z} e^{G z^{2} / 2}\right)$ with $\mathcal{A}=V_{i}, A_{i}$. If the behavior of $\Phi=M z^{2}$ were maintained, the Regge slope would be
$4 \sqrt{G^{2}+q^{2} M^{2}}$. However, it cannot be so; the equation for the chiral scalar $\Phi$ in the presence of $\varphi$ is

$$
-\Phi^{\prime \prime}(z)+\left(\varphi^{\prime}(z)+\frac{3}{z}\right) \Phi^{\prime}-4 \frac{\Phi}{z^{2}}=0 .
$$

Notice that the behavior of $\Phi$ in large $z$ is dominated by $\varphi=G z^{2}$, because $\varphi^{\prime} \gg 3 / z$ there. Therefore, asymptotic behavior of $\Phi$ is either $\Phi \simeq \exp \left(G z^{2}\right)$ or $\Phi \simeq M_{1} \exp \left(-1 / G z^{2}\right)$ with dimensionless parameter $M_{1}$. Since we should take the finite solution, we have

$$
\Phi \simeq M_{1} \text { for } z \rightarrow \infty .
$$

For large quantum number $n$,

$$
m_{n}^{2}=G\left(4 n+2 \sqrt{1+M_{1}^{2}}+2\right) .
$$

For small $n$, configuration of $\Phi$ can make a small nonlinear component to the trajectory.

Notice that the chiral symmetry breaking, although its breaking is spontaneous, does not contribute to the Regge slope, so that the Regge slope is determined only by the scale symmetry breaking scale. Indeed, the Regge slopes of all the meson family are the same, and we point out that this makes the soft-wall model explain why this is so. The chiral symmetry breaking contributes to the Regge intercept by the parameter $M_{1}$, which is expected to be zero in the limit of chiral symmetry restoration. This explains why the vector and axial vector mesons will be the same, which is another phenomenological fact.

In summary, both the chiral symmetry breaking and nontrivial dilaton configuration discussed in this section are natural ways to introduce a physical scale. The issue here was whether two mechanism can coexist or compete. For the former case, we would have two independent scales in QCD. What we found here is that interestingly they compete and only one mechanism survives, and as a consequence, we have only one scale.

\section{OTHER MODELS WITH LINEAR CONFINEMENT}

In the rest of this paper, we provide other models without the overall dilaton factor $e^{-\varphi}$ yet having linear Regge trajectories for the future model building for QCD and condensed matter. In the confined phase, we should treat the particle of each spin individually,

$$
S=\sum_{s \geq 2} S_{V, s}+S_{A, s}
$$

where index $s$ is for spin $s$.

The vector meson cannot couple to $\Phi$ because $\Phi$ does not have the vector charge. Usually, the dilaton, the Goldstone 
boson of the scale symmetry, is introduced as a real massless scalar which is dual to the gluon operator $\operatorname{Tr} G_{\mu \nu}^{2}$ whose nonzero vacuum expectation value breaks the scale symmetry. For our purpose, we identity it as a square root of the gluon operator. It should couple to the vector meson; otherwise, the latter will be massless. The action of the vector meson is $S_{V}=\int d^{d+1} x \sqrt{-g} \mathcal{L}_{V}$ with

$$
\mathcal{L}_{V}=-\frac{1}{4} F_{V}^{2}-\frac{1}{2} \nabla_{\mu} \varphi \nabla^{\mu} \varphi-g^{2} \varphi^{2} V_{\mu} V^{\mu}
$$

Our dilaton has following solution $\varphi=G z^{2}$ as before. The equation for the transverse vector meson is still given by the Schrödinger equation (7) with $q M$ replaced by $g G$. Therefore, the spectrum of the vector meson is again a linear tower given by

$$
m_{n, \text { vector }}^{2}=4 g G(n+d / 4) .
$$

If we have added the $-m_{V}^{2} V_{\mu_{1} \cdots \mu_{s}}^{2}$ term to the Lagrangian of the spin $s$ vector meson, the spectrum would change to

$$
m_{s, n}^{2}=2 g G\left(2 n+p_{V}+1\right),
$$

with $p_{V}=2 \sqrt{\left(s-1+\frac{d-2}{4}\right)^{2}+m_{V}^{2}}$. To fit the data for the $\rho$ meson with $d=4, s=1$, we can take $p \simeq-1$, which can be done most naturally by setting $m_{V}^{2}=0$. That is, for the phenomenology, it is better not to introduce the bulk mass of the vector meson.

\section{A. Gluon condensation and axial mesons}

The anomaly of the $U(1)_{A}$ can be considered as a part of the spontaneous breaking of the axial symmetry, and we should open the possibility that the promoted bulk gauge invariance can be broken explicitly at the bulk level, because the bulk theory should include the quantum dynamics of the boundary theory at the classical level. This implies that axial symmetry could have been further broken by adding the bulk mass term $-m_{A}^{2} A_{\mu_{1} \cdots \mu_{s}}^{2}$ to the Lagrangian of the spin $s$ axial vector meson.

Then, the spectrum would change to

$$
m_{s, n}^{2}=2 q M\left(2 n+p_{A}+1\right),
$$

with $p_{A}=2 \sqrt{\left(s-1+\frac{d-2}{4}\right)^{2}+m_{A}^{2}}$. Again, when one considers the equation of motion in the Schradinger form, it becomes similar to that of the model having the dilaton soft-wall model, and according to Refs. [9,10], $4 q M=$ $1.25(\mathrm{GeV})^{2}$ and $m_{A}=0.5$ can fit the data well.

It could have been broken even further by dilaton coupling $-\varphi A_{\mu} A^{\mu}$. However, then the spectrum is changed by $q M \rightarrow \sqrt{(q M)^{2}+(g G)^{2}}$ so that the slope of Regge trajectory of the axial vector is bigger than that of vector meson, which is not consistent with the data in Ref. [9].
Therefore, we do not add the dilaton coupling of the axial meson. To make two slopes equal, we need

$$
g G=q M .
$$

However, this is not a good consequence for the QCD, because it means a fine-tuning is necessary for the universality of the Regge slope.

\section{B. Glueball spectrum}

To understand the color confinement, it is good idea to look at the behavior of a gauge invariant version of color fields, say $\mathcal{O}=\operatorname{Tr}\left(G_{\mu \nu}\right)^{n}$, under the gluon condensation. Let $\phi$ be the scalar field in the bulk which is dual to the scalar field $\mathcal{O}$ of dimension $\Delta$. Then, the dynamics of $\phi$ "inside" the bag can be studied by

$S_{\phi}=\frac{1}{2} \int d^{5} x \sqrt{-g}\left(-\nabla^{\mu} \phi \nabla_{\mu} \phi-m^{2} \phi^{2}-g_{S}^{2} \varphi \phi^{2}\right)$.

Using the solution $\varphi=G^{2} z^{4}$ as before, the Schrödinger form of the scalar equation is given by Eq. (7) with $p_{S}^{2}=m^{2}+4$, and the scalar meson spectrum is given by

$$
m_{n, \text { scalar }}^{2}=2 g_{S} G\left(2 n+p_{S}+1\right) .
$$

The linear spectrum of the glueball is interesting, but what is more important for us here is the behavior of the wave function equation (8), which says that the color flux outside the bag, $z>z_{m}$, is exponentially suppressed, proving the color confinement within the bag under the presence of the gluon condensation.

Notice that in many of our models, we need to choose the bulk mass of the theory properly to get the promised combination $n+s$. That spin dependent mass is necessary for the spectral formula has been known from the original paper [7] but has not been clear so far. Notice that string theory encodes all the spin simultaneously, while in field theory, the action for each spin should be considered one by one. Now, how do we add up such spin dependent field theories to describe the holographic image of the bulk fundamental string? While the kinetic terms are canonical, it is not surprising to have ambiguities in the mass term of spin $s$ excitation. We suggest that reproducing the linear spectrum can be used as a guiding principle to determine them.

\section{CONCLUSIONS}

We finish the paper with a summary and a few remarks. First, one may ask the problem of divergence of the scalar solution in the IR region $(z \rightarrow \infty)$. This is precisely the problem of the probe approach where we assume that the gravity background is fixed as AdS. There is a known resolution to this: in reality, the backreaction of AdS will either create a horizon, a natural IR cutoff, or smooth out 
the solutions. Whether the probe solution is useful or not depends on what we do with it. If we evaluate the thermodynamic quantity, we would fail. But for the spectrum, the background will be useful because the true solution will be similar to the probe solution away from the singular region $(z \rightarrow \infty)$, which is forbidden for the wave function of excitations anyway: we are looking for the equation of motion of the vector field's perturbation, which was shown to be written (after a change of variable) as a Schrödinger equation whose potential contains the $z^{2}$ term with $z=1 / r$. Such a configuration provides a soft wall, providing the barrier so that the wave function will not penetrate to the IR region $z \rightarrow \infty$. This is an effective way to cut out the IR regime and justify the use of the probe solution for the problem of spectrum. One can show that when we consider the backreaction, a horizon is developed, and as the horizon grows, the potential's $z^{2}$ regime will retract. The potential will not grow like $z^{2}$ indefinitely but collapse to $-\infty$ at the horizon, so that the higher quantum number of the linear spectrum will be deformed and disappear. Details of such an effect are a complicated correction to the simple phenomena describe here.

Next, in a theory where the dilaton is not involved, chiral symmetry breaking can contribute to the slope of the axial vector meson. But when scale symmetry breaking comes with the coupling of overall $e^{-\varphi}$ coupling, like the soft-wall model, the theory changes its face: the chiral symmetry breaking effect is eaten by that of the scale symmetry breaking and does not contribute to the Regge slope. However, the latter can contribute to the intercept of the Regge trajectory and the mass of the axial vector meson.

We identified the origin of the Regge slopes as the condensation of the order parameter that controls the symmetry breaking scales. The linearity of the Regge trajectory is generated because the potential is the same form as that of a three-dimensional isotropic harmonic oscillator where $z$ play the role of the radial coordinate. The $\frac{1}{z^{2}}$ term is due to the confining gravity of the AdS space, while the quadratic potential is by gluon and chiral condensations. The latter provides an infinite "soft wall," and it can be attributed as a property of the vacuum with such condensation. Our results suggest that the color confinement and the Regge slope are consequences of gluon condensation. Therefore, by measuring the Regge slopes, we can determine gluon condensation, but we cannot determine the chiral condensation so easily.

We now comment on the points where our work has a loose end. We studied a solution to the field equations in the probe limit, and while this solution does not obey the standard infrared boundary condition in AdS/CFT, perhaps there is a more complicated version of the model (where one can speculate on a few different ways to do this) in which this approximate solution is the preferred one up to very close to the horizon where the probe limit breaks down.

A related point that requires future work is the dynamical condensation of the scalar. It means that the latter is determined as a function of other inputs like density and temperature. Setting up such dynamical generation requires the coupling of the scalar to other fields that already contain a scale, and the presence of the horizon. Then, two boundary conditions can be chosen as a sourceless condition at the boundary and regularity condition at the IR horizon; then, the condensation is determined from these two data and the scale involved in the fields that is coupled to the scalar. Such a process is preferable for many purposes like in the theory of holographic superconductivity where the condensation was determined in terms of the temperature and chemical potential, although it is not always necessary; we could equally well determine the chemical potential in terms of the temperature and condensation. That is, the condensation can be an equally good input of the theory as in the original AdS/CFT setup where we determine other quantities like the spectrum in terms of the condensation. In the original soft-wall model paper [7], it was argued that the dilaton configuration would be induced due to the instability of tachyon condensation, which corresponds to the gluon condensation from the boundary point of view. Here, we are considering the Abelian Higgs model where the scalar is the dual of the boundary fermion bilinear $\bar{\psi} \psi$ so that the scalar condensation represents the "chiral" condensation. This is exactly parallel to the gluon condensation to dilaton based soft-wall model, and in both cases, condensation should invoke a new vacuum through the instability of the AdS vacuum.

\section{ACKNOWLEDGMENTS}

We thank Youngman Kim, Yongseok Oh, Junchen Rong, and especially to Mannque Rho for useful discussions. This work is supported by Mid-Career Researcher Program through the National Research Foundation of Korea Grant No. NRF-2016R1A2B3007687. We are also grateful for the support of Asia Pacific Center for Theoretical Physics (APCTP) during the focus workshop "holography and geometry of quantum entanglement".

\section{APPENDIX: MODELS WITH HIGHER RANK TENSORS}

In the first two subsections of this Appendix, we study models with diffeomorphism invariance but without gauge invariance. In the final subsection, we study the theory with gauge invariance as well as diffeomorphism invariance.

\section{Rank-s totally antisymmetric tensor without gauge symmetry}

We may start with field equation

$$
\begin{aligned}
& \frac{1}{\sqrt{-g}} \partial_{\mu}\left(\sqrt{-g} g^{\mu \nu} g^{\alpha_{1} \beta_{1}} \ldots g^{\alpha_{s} \beta_{s}} \partial_{\nu} A_{\beta_{1} \ldots \beta_{s}}\right) \\
& =\left(\Phi^{2}+m_{A}^{2}\right) g^{\alpha_{1} \beta_{1}} \ldots g^{\alpha_{s} \beta_{s}} A_{\beta_{1} \ldots \beta_{s}} .
\end{aligned}
$$


With axial gauge choice $A_{z x_{1} \ldots x_{s}}=0$, the equation of motion of $A_{x_{1} \ldots x_{s}}:=B e^{i(k \cdot x-\omega t)}$ takes the form

$$
-z^{-\alpha} \partial_{z}\left(z^{\alpha} \partial_{z} B\right)+\left(\Phi^{2}+m_{A}^{2}\right) z^{-2} B=m_{n}^{2} B,
$$

where $\alpha=-d+2 s+1$ and $m_{n}^{2}=\omega^{2}-k^{2}$. Using the identity

$$
\partial_{z}\left(z^{\alpha} \partial_{z} B\right)=z^{\alpha / 2}\left(\phi^{\prime \prime}-\frac{\left(\frac{\alpha-1}{2}\right)^{2}-\frac{1}{4}}{z^{2}} \phi\right)
$$

with $B=z^{-\alpha / 2} \phi$, we get

$$
\begin{gathered}
-\phi^{\prime \prime}+\left(\frac{p^{2}+m_{A}^{2}-\frac{1}{4}}{z^{2}}+M^{2} z^{2}\right) \phi=m_{n}^{2} \phi \\
E_{n, s}=M\left(4 n+2 \sqrt{p^{2}+m_{A}^{2}}+2\right),
\end{gathered}
$$

with $p^{2}=\left(s-\frac{d}{2}\right)^{2}$. Then, the desired spectrum

$$
E_{n, s}=M(4 n+4 s-4+d)
$$

can be obtained for $m_{A}^{2}=3(s-1)(s+d-3)$.

\section{Rank-s totally symmetric tensor without gauge symmetry}

For the same gauge choice and the variable, the equation of motion is

$$
V(z)=\frac{\left(\frac{d}{2}\right)^{2}+s-\frac{1}{4}+m_{A}^{2}}{z^{2}}+M^{2} z^{2} .
$$

The spectrum

$$
E_{n, s}=M(4 n+4 s+d-4)
$$

can be obtained if $m_{A}^{2}=(2 s-3)(2 s-3+d)-s$.

If there were overall dilaton factor $e^{\varphi}$ with $\varphi=M z^{2}$ in the action,

$$
V(z)=\frac{\left(\frac{d}{2}\right)^{2}+s-\frac{1}{4}+m_{A}^{2}}{z^{2}}+M^{2} z^{2}+d-2,
$$

then we can have

$$
E_{n, s}=M(4 n+4 s-4+d),
$$

by choosing $m_{A}^{2}=4 s^{2}-9 s+4-d^{2} / 4$.

\section{Higher spin theory}

In Ref. [7], the rank-s totally symmetric tensor with gauge symmetry $A_{\mu_{1} \ldots \mu_{s}} \rightarrow A_{\mu_{1} \ldots \mu_{s}}+\nabla_{\left(\mu_{1}\right.} \xi_{\left.\mu_{2} \ldots \mu_{s}\right)}$ was identified as the spin $s$ theory. The residual gauge transformation which leaves $A_{z \mu_{2} \ldots \mu_{s}}$ invariant [7] is determined by

$$
\nabla_{\left(\mu_{1}\right.} \xi_{\left.\mu_{2} \ldots \mu_{s}\right)}=0 .
$$

Using $\Gamma_{z \mu}^{\mu}=-1 / z, \Gamma_{i i}^{z}=1 / z, \Gamma_{t t}^{z}=-1 / z$, we get

$$
\partial_{z} \xi+\frac{2 s-2}{z} \xi=0
$$

namely, $z^{2 s-2} \xi_{x}(z, \mathbf{x}):=\tilde{\xi}_{x_{1}, \ldots, x_{s}}(\mathbf{x})$ is $z$ independent. Introducing the scaled variable $\tilde{A}_{x_{1}, \ldots, x_{s}}:=z^{2 s-2} A_{x_{1}, \ldots, x_{s}}$, the residual gauge transformation in terms of the tilde variable is nothing but the shifting: $\tilde{A}_{x_{1}, \ldots, x_{s}} \rightarrow \tilde{A}_{x_{1}, \ldots, x_{s}}+$ $\tilde{\xi}_{x_{1}, \ldots, x_{s}}$. The action can be written as

$$
S=\int z^{\alpha} e^{\varphi}\left[\left(\partial_{\mu} \tilde{A}_{x_{1}, \ldots, x_{s}}\right)^{2}\right]
$$

where with $\varphi=M z^{2}$ and $\alpha=-(1+d)+2(s+1)-2(2 s-2)=$ $4-d+1-2 s$. In other words, the action should be designed such that Eq. (A13) holds using covariant derivatives. Now, using the methods which are by now familiar, we have

$$
E_{n, s}=M(4 n+4 s-4+d),
$$

as was described in Ref. [7] for $d=4$.

One should notice that the mass term is not invariant, and therefore the invariance under the residual gauge transformation should determine the mass [7] to be $m_{A}^{2}=$ $s^{2}-s-4$. For the same reason, the naive scalar coupling term such as $\Phi^{2}\left(\tilde{A}_{x_{1}, \ldots, x_{s}}\right)^{2}$ is not allowed.
[1] G. Veneziano, Construction of a crossing-symmetric, Regge behaved amplitude for linearly rising trajectories, Nuovo Cimento A 57, 190 (1968).

[2] S. Mandelstam, Vortices and quark confinement in nonabelian gauge theories, Phys. Rep. 23, 245 (1976).
[3] J. M. Maldacena, The large N limit of superconformal field theories and supergravity, Int. J. Theor. Phys. 38, 1113 (1999); Adv. Theor. Math. Phys. 2, 231 (1998).

[4] E. Witten, Anti-de Sitter space and holography, Adv. Theor. Math. Phys. 2, 253 (1998). 
[5] S. S. Gubser, I. R. Klebanov, and A. M. Polyakov, Gauge theory correlators from noncritical string theory, Phys. Lett. B 428, 105 (1998).

[6] J. Erlich, E. Katz, D. T. Son, and M. A. Stephanov, Qcd and a Holographic Model of Hadrons, Phys. Rev. Lett. 95, 261602 (2005).

[7] A. Karch, E. Katz, D. T. Son, and M. A. Stephanov, Linear confinement and AdS/QCD, Phys. Rev. D 74, 015005 (2006).
[8] D. B. Kaplan, J.-W. Lee, D. T. Son, and M. A. Stephanov, Conformality lost, Phys. Rev. D 80, 125005 (2009).

[9] A. V. Anisovich, V. V. Anisovich, and A. V. Sarantsev, Systematics of q anti-q states in the $\left(n, M^{2}\right)$ and $\left(J, M^{2}\right)$ planes, Phys. Rev. D 62, 051502 (2000).

[10] S. He, M. Huang, Q.-S. Yan, and Y. Yang, Confront holographic QCD with Regge trajectories, Eur. Phys. J. C 66, 187 (2010). 